Chirurg 2009 $\cdot 80: 767$

DOI 10.1007/s00104-009-1783-z

Online publiziert: 14. August 2009

(c) Springer Medizin Verlag 2009

J.R. Siewert • Universitätsklinikum Heidelberg

\title{
Nicht die postoperative Komplikation ist das Problem, sondern der Umgang mit ihr!
}

\section{Noch ein Wort zu den entstehenden Kosten}

griffsimmanent und unvermeidbar; nach dem Motto: „Wo genäht wird, gibt es auch Insuffizienzen“. Natürlich wird der erfahrene Chirurg sein Berufsleben lang daran arbeiten, möglichst alle Komplikationen $\mathrm{zu}$ vermeiden, dennoch werden sie auch ihn ereilen. Es ist also klüger, sich den Komplikationen zu stellen, nach ihnen diagnostisch zu fahnden und ihre möglichst frühzeitige Therapie anzustreben. „If you can't beat him, join him."

Interessanterweise sind die postoperativen Komplikationen offenbar nicht unter eine Schwelle von ca. 15-20\% zu drücken. Ebenso klar ist, dass $80 \%$ dieser postoperativen Komplikationen eingriffsspezifisch, d.h. chirurgischer Natur sind. Sogenannte allgemeine schicksalhafte Komplikationen (Pneumonie, Lungenembolie etc.) sind heute dagegen eher selten. Auch hier gilt es, sich den chirurgischen Komplikationen zu stellen und mit ihnen adäquat umzugehen, anstatt nach raren allgemeinen Komplikationen zu fahnden und nach dem Motto: „Was nicht sein darf, kann nicht sein“ zu verfahren.

Intensivüberwachung und die Möglichkeit der modernen Bildgebung ermöglichen es heute, postoperative Komplikationen rechtzeitig zu identifizieren und eine geeignete Therapie einzuleiten. Hier gilt es: „Daran denken und sich dazu bekennen“.

Die Komplikationen als solche sind nicht nur für den Patienten gefährlich und für den Operateur unangenehm; sie haben auch weitere Konsequenzen für den Verlauf und die Prognose. Die Prognose des individuellen Patienten wird langfristig über die postoperative Gefährdung hinaus - schlechter, d.h. postoperative Komplikationen sind als unabhängiger Prognosefaktor zu sehen.
Eine postoperative Komplikation verlängert die Aufenthaltsdauer auf der Intensivstation um den Faktor 4. Die Krankenhauskosten steigen damit an; so ist in den USA errechnet worden, dass allein jede pulmonale Komplikation Extrakosten von 52.00o US-Dollar nach sich zieht. Andreas Tecklenburg weißt in seinem Leitthemenbeitrag zur betriebswirtschaftlichen und volkswirtschaftlichen Problematik postoperativer Komplikationen in diesem Heft auf die hohen sog. „Ausreißerkosten“ hin, die sich in großen Kliniken der Maximalversorgung leicht auf Millionenbeträge addieren lassen.

\section{Eine weitere Frage bedarf der Beantwortung}

Warum sind die postoperativen Ergebnisse in den sog. "highvolume hospitals“ besser? Nicht weil die Komplikationsrate als solche geringer wäre, sondern weil man dort im Umgang mit postoperativen Komplikationen trainierter und erfahrener ist. Entscheidend ist eben nicht die Komplikation, sondern der Umgang mit ihr. „Highvolume hospitals“ haben auch größere Erfahrungen im Komplikationsmanagement und sind damit erfolgreicher im Umgang mit postoperativen Komplikationen.

\section{Ein letzter Aspekt}

Kann man komplikationsgefährdete Patienten präoperativ erkennen und sie somit gar nicht erst der Gefährdung durch den Eingriff aussetzen? Bartels weist in seinem Beitrag nachdrücklich auf die Bedeutung einer präoperativen Risikoanalyse in der
Chirurgie des oberen Gastrointestinaltrakts hin.

Ganz aktuell hat das American College of Surgery einen sog. „Morbidity and Mortality Risk Calculator for Colorecal Surgery“ veröffentlicht (Cohen ME et al. J Am Coll Surg 2009).

\section{— „Es geht also!“ Patientenselektion hilft postoperative Komplikationen zu vermeiden.}

Im vorliegenden Heft von „Der Chirurg“ werden die typischen postoperativen Komplikationen aus Sicht der verschiedenen operativen Disziplinen dargestellt und der Umgang mit ihnen diskutiert. Die eingeladenen Autoren haben jeweils große Erfahrung auf ihrem Fachgebiet, sowohl in der Vermeidung von Komplikationen als auch im Komplikationsmanagement. Sie geben ihre Erfahrungen hier weiter. Nutzen sie diese Erfahrungen.

Um es noch einmal klarzustellen: Nicht die Komplikation ist das Problem, sondern der Umgang mit ihr! Noch zu viele Chirurgen haben im Umgang mit den postoperativen Komplikationen das Diktum im Kopf: „Was nicht sein darf, kann nicht sein“. Dieses Diktum muss geändert werden: „Was sein kann, passiert auch; man muss sich dem Problem stellen.“

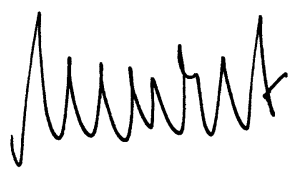

Prof. Dr. med. Dr. h.c. J. Rüdiger Siewert

\section{Korrespondenzadresse}

Prof. Dr. Dr. h.c. J.R. Siewert

Universitätsklinikum Heidelberg Im Neuenheimer Feld 672, 69120 Heidelberg

JR.Siewert@med.uni-heidelberg.de 